\title{
Anti Newton - Refutation of the Constancy of Newton's Gravitational Constant Big G
}

\author{
Ilija Barukčićí ${ }^{*}$ \\ ${ }^{1}$ Horandstrasse, DE-26441 Jever, Germany. \\ * Corresponding author. email: Barukcic@t-online.de \\ Manuscript submitted December 12, 2014; accepted March 13, 2015. \\ doi: 10.17706/ijapm.2015.5.2.126-136
}

\begin{abstract}
In general, the value of Newton's gravitational constant big G has one of the longest history of measurements in physics, but a definite value is still not in sight. Surprisingly, in spite of the fundamental importance of the Newton's universal gravitational constant big G, the more the precision of measurements of Newton's constant big G increased, the more the disparity of the measured value of Newton's constant big G increased. Different groups of experimentalists are repeatedly obtaining conflicting results for the exact value of Newton's constant big G. Is this high degree of uncertainty of Newton's constant big G a result of experimental errors or is the same at the end not a constant at all? The purpose of this publication is to refute the constancy of Newton's constant big G by the proof that Newton's gravitational constant big G is not a constant.
\end{abstract}

Key words: Quantum mechanics, special and general relativity theory, unified field theory, causality.

\section{Introduction}

In 1686 Isaac Newton (1642-1727) introduced his simple and well known universal law of gravitation [1], which states that two object with a certain mass $\left(\mathrm{m}_{0}\right.$ and $\mathrm{M}_{\mathrm{R}}$ ) attract each other with a certain force $\mathrm{F}_{0}$ which is at the end proportional to the product of these masses $\left(m_{0} \times M_{R}\right)$ and the inverse square of the distance $\left(\mathrm{d}_{0}{ }^{2}\right)$ between these masses and a constant [1] of proportionality, called Newton's gravitational constant big G. In 1798, Henry Cavendish [2] and co-workers where the first who were able to obtain a value for Newton's constant big G, using a torsion balance invented by the geologist Rev. John Michell, as $6.67 * 10^{-11}\left[\mathrm{~m} 3 /\left(\mathrm{kg} \times \mathrm{s}^{2}\right)\right]$. Newton's gravitational constant big G is part of Einstein's theory of general relativity too. In Einstein's theory of general relativity, developed early in the 20th century, the curvature of space-time is at the end proportional to Newton's gravitational constant big G too.

However, it is worth mentioning that it is difficult to measure Newton's constant big G with great accuracy. Even under the most optimistic conditions, there is still a great uncertainty in the measurement of this constant. The more the precision of measurements of Newton's constant big G increased, the more the disparity between the values of Newton's constant big G increased. Repetitive measurements of the gravitational constant big $\mathrm{G}$ show that big $\mathrm{G}$ varies significantly.

Measurements of Newton's gravitational constant big G below the surface of the earth (in mine shafts) [3], [4], boreholes [5], the deep ocean [6] and in an Airy-type geophysical experiment conducted in a 2-km-deep hole in the Greenland ice cap [7] at depths between 213 and $1673 \mathrm{~m}$ consistently showed significant deviations of Newton's gravitational constant big G from predictions based on Newton's law of gravitation. 
In 2002, Newton's gravitational constant big G was measured around the clock for seven months, using two independent methods. These experiments were carried out using a torsion balance (two small masses of about $0.9 \mathrm{~g}$ and one large mass of about $4.3 \mathrm{~kg}$ ). This first systematic study [8] of changes in Newton's gravitational constant big $\mathrm{G}$ at different times of day and night found a clear daily rhythm.

Between 1983 and 2000, the lowest (1983) average value of Newton's constant big G which can be found in literature is about 6.575 [9], and the highest (2000) average value of Newton's gravitational constant big $\mathrm{G}$ was 6.674215 [10], a 1.486541863 percent difference, too much for a constant.

Today's physics and especially Einstein's theory of general relativity is based on the assumption that some certain fundamental features of nature are constant. In particular, Newton's gravitational constant big $\mathrm{G}$ is supposed to be changeless. Are the laws of nature fixed forever, what if Newton's gravitational constant big $\mathrm{G}$ really does change?

\section{Material and Methods}

Isaac Newton's masterpiece Philosophiaenaturalis principia mathematica was published in the year 1686. Because of the extent to which Newton's Principia has influenced the development of modern science, physics and astronomy, the same work is still of historical importance.

\subsection{Newton's Universal Law of Gravitation}

Isaac Newton (1642-1727) introduced on 5 July 1686 his simple and well known universal law of gravitation in his work PhilosophiaeNaturalis Principia Mathematica. In the Principia (Prop. LXXVI. Theor. XXXVI) on page 198, Newton defines the force of gravity in the following way (see Table 1):

Table 1. Newton and the Gravitational Constant Big G

"visattractivapuncticujusq; decrescit in duplicatarationedistantiæcorporisattracti: dico quod vis tota qua hujusmodiSphæraunaattrahitaliam sit

reciproceproportionalisquadratodistantiæcentrorum." [11]

Translated from the Latin into English.

$<$ the attractive force of every point decreases in the duplicate ratio of the distance of the body attracted; I say, that the whole force with which one of these spheres attracts the other will be reciprocally proportional to the square of the distance of the centres.>

For our purposes, the key word here is proportional. Newton's statement of proportionality is the source of Newton's gravitational constant big G, known to physicists as big G. Newton's law of universal gravitation, from the standpoint of a stationary observer (R), written as an equation in scalar form states

$$
F_{R}=G_{R} \times \frac{m_{R} \times M_{R}}{d_{R}^{2}}
$$

where $F_{R}$ is the force due to gravity between the two masses, $G_{R}$ is the gravitational constant, $m_{R}$ is the first mass, $M_{R}$ is invariant mass which is the same quantity in all inertial frames of reference and for all observers (an observer-independent quantity). Thus far, $d_{R}$ is the distance between the centers of the two masses $m_{R}$ and $M_{R}$.

\subsection{Einstein's Special Theory of Relativity}

The relative motion between two inertial frames of reference, where Newton's laws are valid, causes observers in those frames of reference to measure different values of the particle's energy (mass) and time. Especially, the notion "relativistic mass" $m_{R}$ of an object (i. e. measured by a stationary observer $\mathrm{R}$ ) and the 
time is dependent on the relative velocity $v$ of the observer and depends at the end on the observer's frame of reference. Under circumstances where the relative velocity between two inertial frames of reference is equal to zero, observers will measure the same values for particle's energy (mass) and time and distances too. Thus far, recall

"Wirdenkenuns ... Uhren, welcherelativzumruhenden System ruhend die Zeit $\mathrm{t}\left[t_{r}\right.$, coordinate time, author], relativzumbewegten System ruhend die Zeit $\tau$ [to, proper time, author] anzugebenbefähigtsind ... Esist also $\tau=\mathrm{t}\left(1-(\mathrm{v} / \mathrm{V})^{2}\right)^{1 / 2} . "[12]$

According to Einstein's special relativity theory, it is [13]

$$
m_{0}=m_{R} \times \sqrt[2]{1-\frac{v^{2}}{c^{2}}}
$$

and equally

$$
t_{0}=t_{R} \times \sqrt[2]{1-\frac{v^{2}}{c^{2}}}
$$

too, where $t_{R}$ is the time as measured by the stationary observer $(\mathrm{R})$ and $t_{0}$ is the time as measured by the moving observer ( 0 ).The elapsed time between two events (time interval) is

$$
\Delta T_{0}=\Delta T_{R} \times \sqrt[2]{1-\frac{v^{2}}{c^{2}}}
$$

while $\Delta T_{0}=\left(\begin{array}{lll}{ }_{2} t_{0} & -{ }_{1} t_{0}\end{array}\right)$ and $\Delta T_{R}=\left(\begin{array}{ll}{ }_{2} t_{R} & -{ }_{1} t_{R}\end{array}\right)$. In general, due to special relativity, a distance measured by light rays by a moving observer is $d_{0}=c \times t_{0}$ and equally the same distance measured by light rays by a stationary observer is $d_{R}=c \times t_{R}$. Thus far, we obtain

$$
\begin{gathered}
t_{0}{ }^{2}=t_{R} \times t_{R} \times \sqrt[2]{1-\frac{v^{2}}{c^{2}}} \times \sqrt[2]{1-\frac{v^{2}}{c^{2}}} \\
d_{0}^{2}=c^{2} \times t_{0}^{2}=c^{2} \times t_{R} \times t_{R} \times \sqrt[2]{1-\frac{v^{2}}{c^{2}}} \times \sqrt[2]{1-\frac{v^{2}}{c^{2}}} \\
d_{0}^{2}=c^{2} \times t_{0}^{2}=d_{R}^{2} \times \sqrt[2]{1-\frac{v^{2}}{c^{2}}} \times \sqrt[2]{1-\frac{v^{2}}{c^{2}}}
\end{gathered}
$$

\subsection{Einstein's Field Equation}

Newton's theory of gravity has been superseded by Einstein's (1879-1955) theory of gravity but continues to be used as an approximation of the effects of gravitation. Leaving aside that Newton's theory of gravity is under dispute, Newton's gravitational constant big Gis regarded as valid and part even of Einstein's general relativity theory [14] too. Einstein's field equations are determined i. e. as 


$$
G_{a e}+\Lambda \times g_{a e}=G \times\left(\frac{4 \times 2 \times \pi_{R}}{c \times c \times c \times c} \times T_{a e}\right)
$$

where $G_{a e}$ denote Einstein tensor, $G$ denotes Newton gravitational constant and is treated as a constant too. Due to Einstein's general theory of relativity, the relationship between curvature and momentum is proportional or constant. Einstein's general relativity theory seems to be the correct theory of gravitation at least at low energies. Theoretically, under extreme conditions (i.e. extreme high energy scale) a variation [15] of Newton's gravitational constant can imply an inconsistency of Einstein's field equations.

\subsection{Definitions}

Let $G_{0}$ denote Newton's "gravitational constant" big $\mathrm{G}$ as measured by a moving observer (0). Let $G_{R}$ denote Newton's "gravitational constant" big $G$ as measured by a stationary observer (R).

Of course, we expect that $G_{0}=G_{R}$ or both observers should obtain the same value for Newton's "gravitational constant" big $\mathrm{G}$, if the same is a constant.

\section{Results}

\subsection{Newton's Gravitational Constant Big G Is a Constant}

\section{Claim.}

Newton's "gravitational constant" big G is a constant. Under some well-defined conditions $(\mathbf{v}=\mathbf{0})$ it is

$$
G_{0}=G_{R}
$$

\section{Proof.}

Let us conduct a thought experiment under conditions of inertial frames of reference to proof whether Newton's gravitational constant big $\mathrm{G}$ is a constant or not.

Thus far, Newton (R) and Einstein (O) are performing some measurements concerning Newton's gravitational constant big $\mathrm{G}$ with the help of Schrödinger's cat (m). Einstein (0) as the moving observer is placed within the inertial frame of reference of Schrödinger's cat and at rest relative to Schrödinger's cat. Newton (R) as the stationary observer is located within the inertial frame of the invariant mass $M_{R}$ (i. e. the invariant mass of a planet) and at rest relative to the invariant mass $M_{R}$. Together with Einstein (0), Schrödinger's cat $(\mathrm{m})$ is located on a vehicle, which can move with constant velocity relative to the invariant mass $M_{R}$ and relative to Newton (R).

Both, Newton (R) and Einstein (O) are in agreement concerning Newton's laws of motion, which are covariant with respect to a transformation between inertial frames of reference. In particular, Newton's laws of motion will preserve their form upon transformation from one inertial reference frame to another.

At this stage we point out that $\mathrm{M}_{\mathrm{R}}$ denotes the mass of a planet, which has the same value in all inertial frames, a mass which is invariant relative to transformations between inertial frames. Newton (R), the stationary observer, is at rest relative to the invariant mass $M_{R}$. The distance between the centers of the two masses measured by light as determined by Newton is $d_{R}=c \times t_{R}$ while $d_{0}=c \times t_{0}$ denotes the distance as determined by Einstein. Einstein ( 0 ) and Newton (R) are at rest relative to each other. Newton determines $\mathrm{F}_{\mathrm{R}}$ as the force due to gravity between two masses $m_{R}$ and $M_{R}$. Einstein himself determines $F_{0}$ as the force due to gravity between the two masses in his inertial frame of reference. The gravitational constant as measured by Newton is denoted by $G_{R}$ while $m_{0}$ is the (rest-) mass of Schrödinger's cat in Einstein's inertial frame of reference and $m_{R}$ denotes the (relativistic) mass of Schrödinger's cat as measured by Newton in his own inertial frame of reference. The following Table 2 illustrates this Gedanken experiment once again. 
Table 2. Experimental Setup I

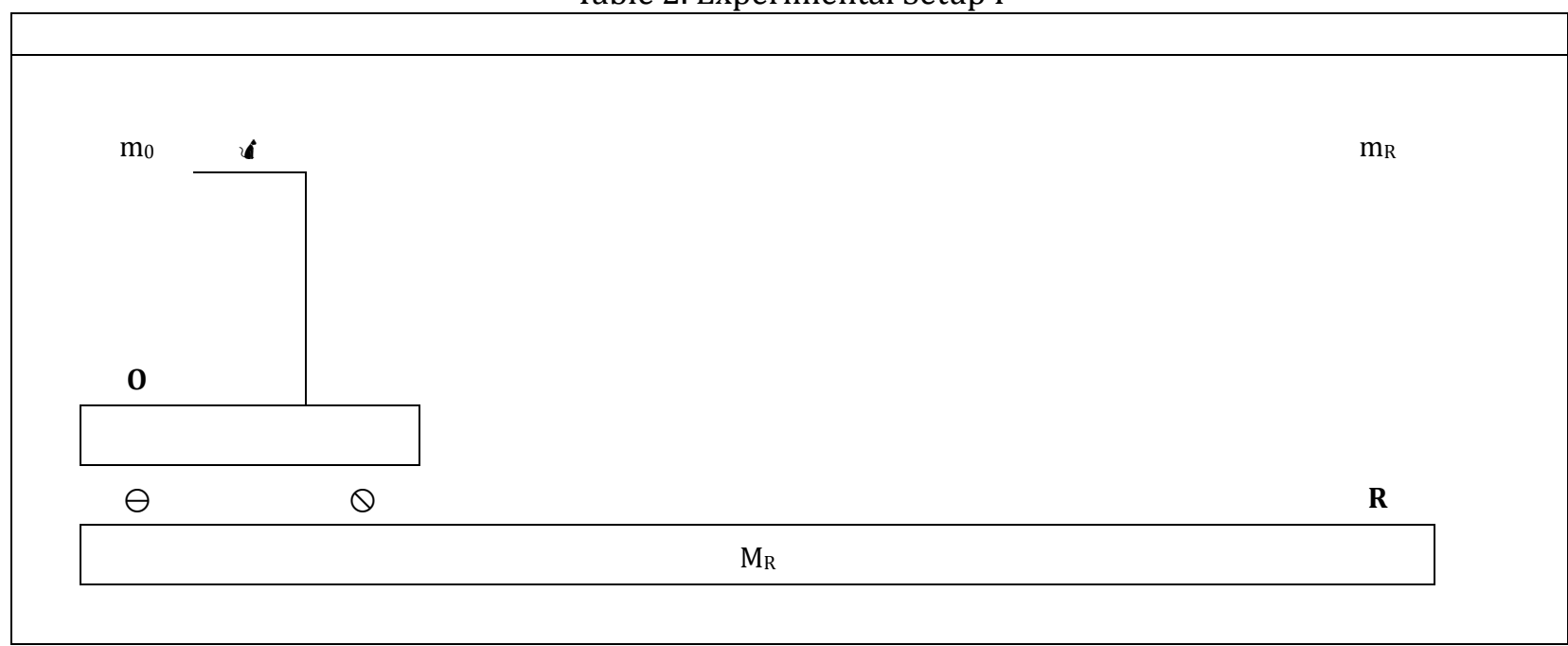

Table 3. Experimental Setup II

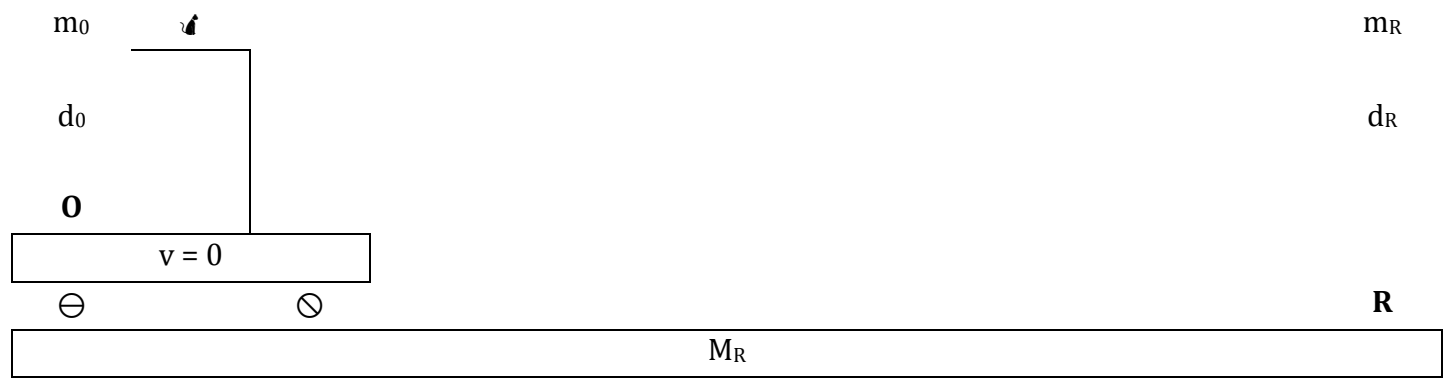

Under these experimental conditions, Newton (R) and Einstein (O) are at rest and not moving uniformly relative to one another. Thus far, no relativity effects must be considered. In general, we obtain (in the scalar form)

$$
F_{0}=G_{0} \times \frac{m_{0} \times M_{0}}{d_{0}^{2}}=G_{R} \times \frac{m_{R} \times M_{R}}{d_{R}^{2}}=F_{R}
$$

Since the relative velocity between Einstein $(0)$ and Newton $(R)$ is equal to zero or $\mathbf{v}=\mathbf{0}$, there is no relativistic mass increase of Schrödinger's cat which must be considered by Newton (R). Further, there is no relativistic time dilation which has to be taken into consideration too. Since $\mathbf{v}=\mathbf{0}$, according to Einstein's special theory of relativity it is

$$
\begin{gathered}
m_{0}=m_{R} \times \sqrt[2]{1-\frac{0^{2}}{c^{2}}} \\
d_{0}^{2}=c^{2} \times t_{0}^{2}=c^{2} \times t_{R} \times t_{R} \times \sqrt[2]{1-\frac{0^{2}}{c^{2}}} \times \sqrt[2]{1-\frac{0^{2}}{c^{2}}} \\
=c^{2} \times t_{0}^{2}=d_{R}^{2} \times \sqrt[2]{1-\frac{0^{2}}{c^{2}}} \times \sqrt[2]{1-\frac{0^{2}}{c^{2}}}
\end{gathered}
$$

or in other words $d_{0}=d_{R}$ and $m_{0}=m_{R}$. Substituting these relationships in the equation above, we obtain 


$$
G_{0}=G_{R}
$$

\section{Q. e. d.}

Under conditions where the relative velocity between inertial frames of reference is equal to zero or where $v=0$ it is not excluded or theoretically it is possible that Newton's gravitational constant big G is a constant.

\subsection{Newton's Gravitational Constant Big G Is Not a Constant}

\section{Claim.}

Newton's "gravitational constant" big G is not a constant. Under some well-defined conditions $(\mathbf{v} \neq \mathbf{0}$, $\mathbf{v}=$ constant) and in contrast to our expectation, it is

$$
G_{0}=G_{R} \times \sqrt[2]{1-\frac{v^{2}}{c^{2}}}
$$

\section{Proof.}

In contrast to our claim, we are of the opinion that Newton's gravitational constant big G is indeed a constant. In the following we will conduct again a thought experiment under conditions of inertial frames of reference where Newton (R) and Einstein $(0)$ are not at rest relative to each other.

Thus far, again Newton (R) and Einstein (O) are performing some measurements concerning Newton's gravitational constant big G with the help of Schrödinger's cat. Again, Einstein (0) as the moving observer is placed within the inertial frame of reference of Schrödinger's cat and at rest relative to Schrödinger's cat. Newton (R) himself is located within the inertial frame of reference of the invariant mass $M_{R}$ (i. e. the invariant mass of a planet) and at rest relative to the invariant mass $M_{R}$. Together with Einstein (0), Schrödinger's cat is located on a vehicle, which can move with constant velocity relative to the invariant mass $\mathrm{M}_{\mathrm{R}}$ and relative to Newton (R).

Under these experimental conditions, Newton (R) and Einstein (0) are moving uniformly relative to one another and performing at the same (period of) time with the help of Schrödinger's cat some measurements concerning Newton's gravitational constant big G.

Under these changed experimental conditions, the inertial frame of reference of Schrödinger's cat together with Einstein $(0)$ is moving with constant velocity relative to Newton's inertial frame of reference. Newton determines $F_{R}$ as the force due to gravity between two masses. Einstein himself determines $F_{0}$ as the force due to gravity between two masses in his inertial frame of reference. Under these experimental conditions, there are no fictitious forces. The gravitational constant as measured by Newton is denoted by $\mathrm{G}_{\mathrm{R}}$ while $\mathrm{m}_{0}$ is the rest mass of Schrödinger's cat in Einstein's inertial frame of reference and $\mathrm{m}_{\mathrm{R}}$ denotes the relativistic mass of Schrödinger's cat as measured by Newton (R), the stationary observer.

At this stage let us point out again that $\mathrm{M}_{\mathrm{R}}$ denotes the mass of a planet, which has the same value in all inertial frames, a mass which is invariant relative to transformations between inertial frames. Newton (R) is at rest relative to the invariant mass $\mathrm{M}_{\mathrm{R}}$. The distance between the centers of the two masses measured by light signals as determined by Newton is $d_{R}=c \times t_{R}$ while $d_{0}=c \times t_{0}$ denotes the distance as determined by Einstein (0). The Table 3 may illustrate this thought experiment (Gedanken experiment) in more detail.

Both masses exert an equal and opposite force on each other (Newton's third law). The force of gravitation due to Newton's universal law of gravitation follows as 


$$
F_{0}=G_{0} \times \frac{m_{0} \times M_{R}}{d_{0}{ }^{2}}=G_{R} \times \frac{m_{R} \times M_{R}}{d_{R}{ }^{2}}=F_{R}
$$

or

$$
G_{0} \times \frac{m_{0} \times M_{R}}{d_{0}^{2}}=G_{R} \times \frac{m_{R} \times M_{R}}{d_{R}^{2}}
$$

Under these experimental conditions relativity effects must be taken into consideration. While $M_{R}$ is treated as invariant mass, due to Einstein's special relativity theory it is

$$
m_{0}=m_{R} \times \sqrt[2]{1-\frac{v^{2}}{c^{2}}}
$$

Table 4. Experimental Setup III

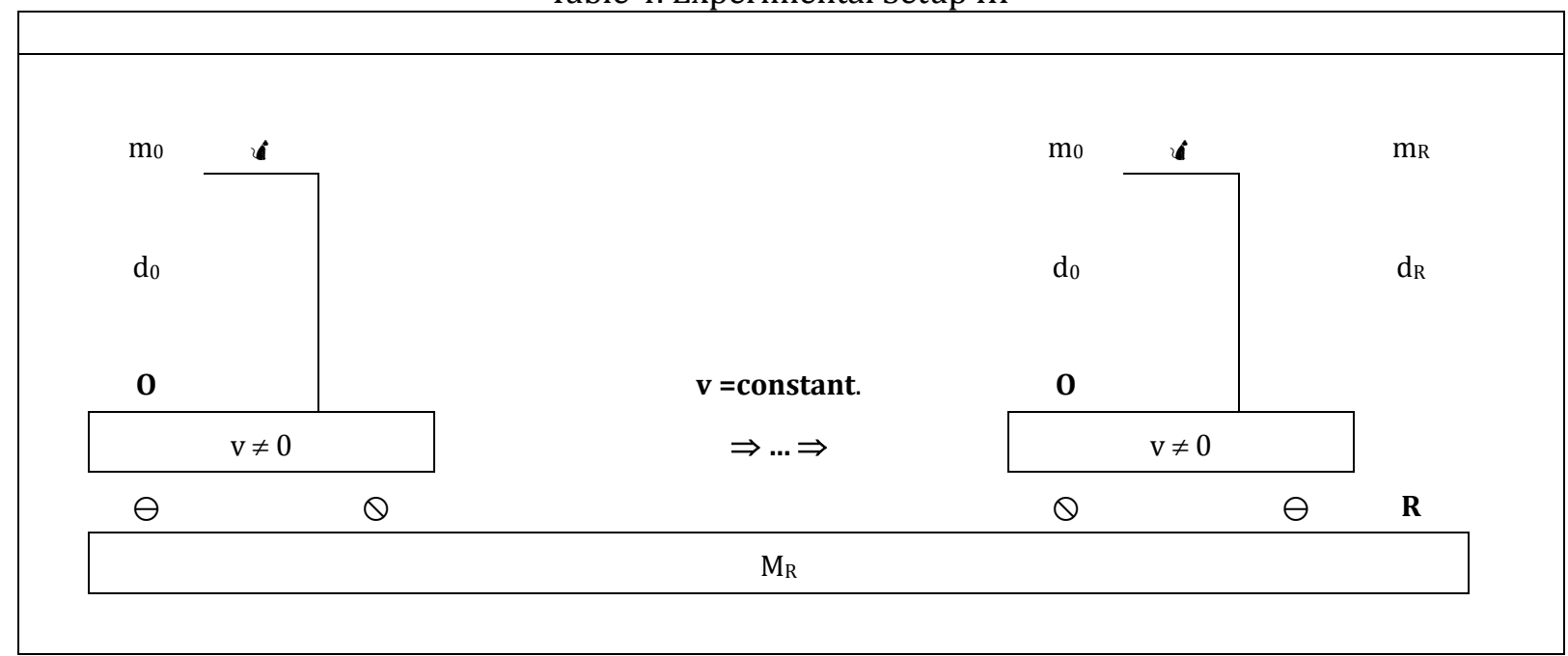

Due to relativity effects, Newton (R), the stationary observer, will measure another relativistic mass $m_{R}$ for Schrödinger's cat than Einstein (0). Substituting (14) into (13), we obtain

$$
G_{0} \times \frac{m_{R} \times \sqrt[2]{1-\frac{v^{2}}{c^{2}}} \times M_{R}}{d_{0}^{2}}=G_{R} \times \frac{m_{R} \times M_{R}}{d_{R}{ }^{2}}
$$

Because of the relativistic time dilation, the distance $d_{0}$ between the center of Schrödinger's cat $\left(m_{0}\right)$ and the invariant mass $M_{R}$ as measured by Einstein (0), the moving observer, using light signals is different from the distance $d_{R}$ between the center of Schrödinger's cat $\left(m_{R}\right)$ and the invariant mass $\mathrm{M}_{\mathrm{R}}$ as measured by Newton (R) himself. Due to Einstein's special relativity theory it is

$$
d_{0}=c \times t_{0}=c \times t_{R} \times \sqrt[2]{1-\frac{v^{2}}{c^{2}}}=d_{R} \times \sqrt[2]{1-\frac{v^{2}}{c^{2}}}
$$

or 


$$
d_{0}=d_{R} \times \sqrt[2]{1-\frac{v^{2}}{c^{2}}}
$$

or

$$
d_{0}^{2}=d_{R}^{2} \times \sqrt[2]{1-\frac{v^{2}}{c^{2}}} \times \sqrt[2]{1-\frac{v^{2}}{c^{2}}}
$$

Substituting (18) into (15) i. e. into Newton's universal law of gravitation, we obtain

$$
G_{0} \times \frac{m_{R} \times \sqrt[2]{1-\frac{v^{2}}{c^{2}}} \times M_{R}}{d_{R}^{2} \times \sqrt[2]{1-\frac{v^{2}}{c^{2}}} \times \sqrt[2]{1-\frac{v^{2}}{c^{2}}}}=G_{R} \times \frac{m_{R} \times M_{R}}{d_{R}^{2}}
$$

Since the most terms cancel out, this equation is equivalent to

$$
\frac{G_{0}}{\sqrt[2]{1-\frac{v^{2}}{c^{2}}}}=G_{R}
$$

\section{Q. e.d.}

Newton's gravitational constant big $G_{R}$ as measured by a stationary observer $(R)$ is different in value from Newton's gravitational constant big $\mathrm{G}_{0}$ as measured by a moving observer (0). This result is a contradiction to our assumption. Our assumption was that Newton (R) and Einstein (0) will measure the same value for Newton's gravitational constant big $\mathrm{G}$ or it has to be that $G_{0}=G_{R}$.

In fact, as soon as relativity effects have to be considered, Newton's gravitational constant big G cannot be regarded or treated as being a constant. About 328 years after introduction, the constancy of Newton's gravitational constant big $\mathbf{G}$ is refuted which has far reaching consequences, especially for Einstein's general theory of relativity and for our understanding of science as such.

\section{Discussion}

Our experimental setup chosen assures conditions of an inertial reference frame or it assured that Newton's first law is valid. That is, an object (i. e. Schrödinger's cat) experiences no net force (a vector quantity) due to other bodies (i. e. the planet $M_{R}$ ). Thus far, the object (i. e. Schrödinger's cat) either remains at rest or remains in motion with constant velocity vin a straight line. Since no net force is acting on the system, the total momentum of the system remains the same. In other words, there is no acceleration (a vector quantity). Einstein's Special Theory of Relativity is valid especially for systems that are not accelerating. The experimental setup before assures conditions where Einstein's Special Theory of Relativity is valid. Thus far, even under conditions of Einstein's special theory of relativity, Newton's gravitational "constant" big G must be a constant, but the same is not.

As previously discussed, measurements of Newton's gravitational "constant" big G consistently showed deviations of Newton's gravitational constant big G from predictions based on Newton's law of gravitation. 
Our proof above demonstrated in a mathematically consistent way, that Newton's gravitational "constant" big $\mathrm{G}$ is not a constant. Only under circumstances where the relative motion between two inertial frames of reference is equal to zero, Newton's gravitational "constant" big G can be regarded as being as constant. But as soon as there is a relative motion between two inertial frames of reference, Newton's gravitational "constant" big G is no longer a constant. At the end, Newton's gravitational "constant" big G depends on observer's frame of reference.

Under some circumstances, the Doppler effect [16], named after the Austrian physicist Johann Christian Doppler (1803-1853), in the relativistic form as introduced by Einstein [17], can be used to determine the value of Newton's "constant" big G of a distant star or Galaxy. However, and for the sake of the argument only, the transverse relativistic Doppler effect is of use too. The transverse relativistic Doppler Effect was derived by Einstein as

"Bezeichnetv dieAnzahl der Schläge der Uhr pro Zeiteinheitfür den ruhenden, $v_{0}$ die entsprechendeAnzahlfür den mitbewegtenBeobachter, so ist" [18].

$$
\frac{v}{v_{0}}=\sqrt{1-\left(\frac{v}{V}\right)^{2}}
$$

Recall, $V=c$. According to Einstein's special relativity theory, the moving light source emitted frequency with respect to the observer is reduced by the Lorentz factor. We rearrange (20). Thus far, it is to be noted that

$$
\frac{G_{0}}{G_{R}}=\sqrt[2]{1-\frac{v^{2}}{c^{2}}}
$$

Equate (21) and (22). Under these conditions it is possible to calculate the numerical value of Newton's gravitational "constant" big $G_{0}$ of our moon, of our sun, of a distant galaxy [19] et cetera using the wavelength measured.

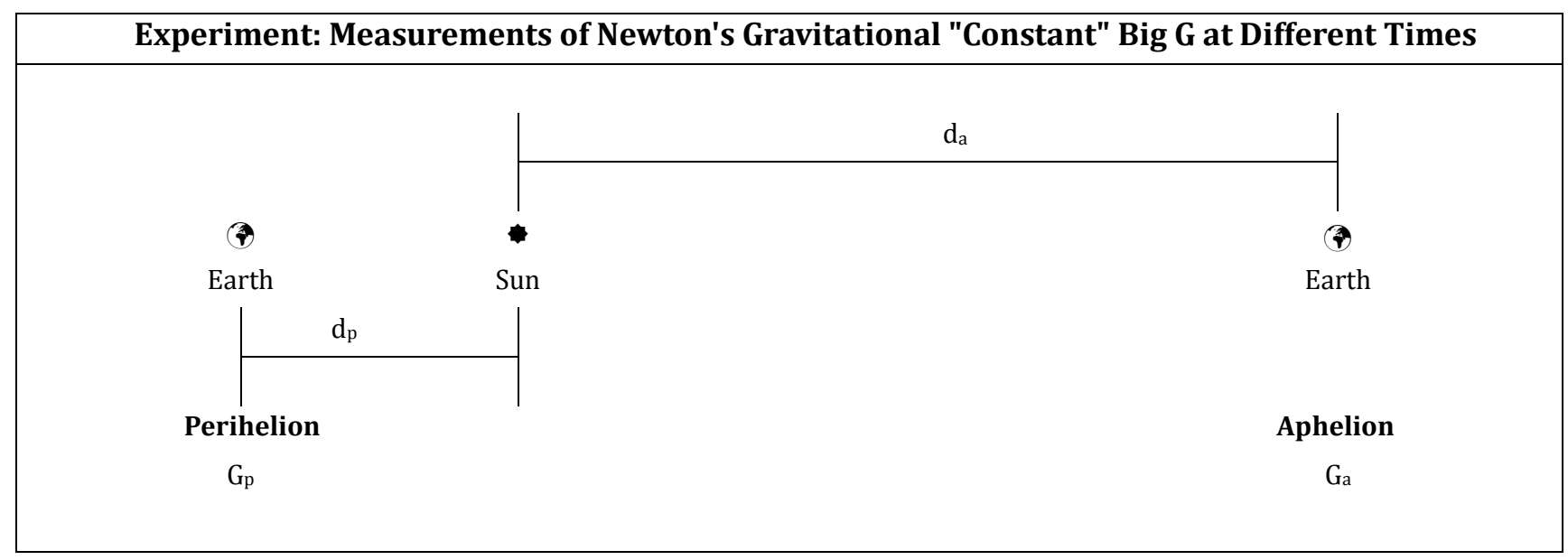

The following experiment can help us to determine definitely and accurately whether Newton's gravitational "constant" big $\mathrm{G}$ is a constant or not.

Measurements of Newton's "constant" big G are performed under "identical" conditions at perihelion and at aphelion. At perihelion the earth (approximately an inertial frame of reference) is closest to our sun and 
has a different orbital velocity relative to the sun than at aphelion (the point in the orbit of our earth, where the earthis farthest from the sun). Under these conditions, the difference in orbital velocity is small but big enough (if the number of experiments $n$ is big enough) to proof whether Newton's "constant" big $G$ is reference frame dependent or not.

A statistically significant difference of the value of Newton's "constant" big $G_{p}$ as measured at perihelion compared to the value of Newton's constant big $G_{a}$ as measured at aphelion cannot be accepted at all, if Newton's constant big G is a "constant".

As derived in (20), it is a straightforward consequence of Einstein's theory of special relativity that Newton's constant big G is not a constant. The one who should be able to make the proof that Newton's constant big $G$ is a constant; the one would have refuted Einstein's theory of special reality which of course is much more than a major undertaking.

\section{Conclusion}

The constancy of Newton's gravitational constant big G is refuted. Newton's gravitational constant big G is not a constant.

\section{References}

[1] Isaac, N. (1686, July 5). PhilosophiaeNaturalis Principia Mathematica. Londini: S. Pepys, Reg. Soc. Praeses. p. 198.

[2] Henry, C. (1798). Experiments to determine the density of the earth. Philosophical Transactions of the Royal Society of London, 88, 469-526.

[3] Tuck, S. C. H. G. (1984). A new mine determination of the Newtonian gravitational constant. Nature, 307, 714-716.

[4] Holding, S. C., Stacey, E. D., \& Tuck, G. (1986). Gravity in mines. An investigation of Newton's law. Physical Review D: Particles and Fields, 33, 3487-3494.

[5] Hsui, A. T. (1987). Borehole measurement of the Newtonian gravitational constant. Science, 237, 881-883.

[6] Stacey, E. D., \& Tuck, G. (1981). Geophysical evidence for non Newtonian gravity. Nature, 292, 230-232.

[7] Ander, M. E., et al. (1989). Test of Newton's inverse-square law in the Greenland Ice Cap. Physical Review Letters, 62,985-988.

[8] Gershteyn, M. L., et al. (2004). Experimental evidence that the gravitational constant varies with orientation. Gravitation \& Cosmology, 8(3), 243-246.

[9] Faller, J. E., \& Koldewyn, W. A. (1983). A prototype measurement of the Newtonian gravitational constant using an active magnetic suspension torsion fiber. Precision measurement and gravity experiment. Proceedings of the 1983 International School and Symposium (pp. 541-556).

[10] Jens, H. G. \& Stephen, M. M. (2000). Measurement of Newton's constant using a torsion balance with angular acceleration feedback. Physical Review Letters, 85(14), 2869-2872.

[11] Isaac, N. (1686, July 5). Philosophiae Naturalis Principia Mathematica. Londini: S. Pepys, Reg. Soc. Praeses.

[12] Albert, E. (1905). Zur elektrodynamikbewegter körper. Annalen der Physik, 322(10), 891-921.

[13] Gilbert, N. L., \& Richard, C. T. (1909). The principle of relativity, and non-Newtonian mechanics. Proceedings of the American Academy of Arts and Sciences (pp. 711-724).

[14] Albert, E. (1916). Die grundlage der allgemeinen relativitätstheorie. Annalen der Physik, 354(7), 769-822.

[15] Brans, C., \& Dicke, R. H. (1961). Mach's principle and a relativistic theory of gravitation. Physical Review, 
124(3), 925-936.

[16] Johann, C. D. (1842). Über das farbige licht der doppelsterne und einigeranderer gestirne des himmels. Abhandlungen der Königl. Böhm. Gesellschaft der Wissenschaften, V(2), 465-482.

[17] Albert, E. (1905). Zur elektrodynamikbewegter körper. Annalen der Physik, 322(10), 891-921.

[18] Albert, E. (1907). Über die möglichkeiteinerneuen prüfung des relativitätsprinzips. Annalen der Physik, 328(6), 197-198.

[19] Lehnert, M. D. et al. (2010). Spectroscopic confirmation of a galaxy at red shift $z=8.6$. Nature, 467(7318), 940-942.

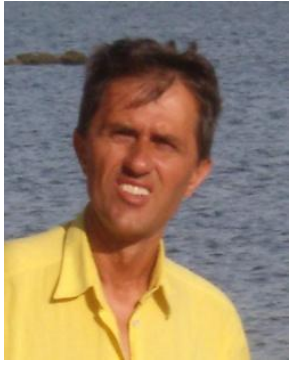

Ilija Barukčić was born on October 1, 1961 in Novo Selo (Bosna i Hercegovina, former Yugoslavia). He studied human medicine (physics and philosophy) at the University of Hamburg from 1982 to 1989. Barukčić graduated in 1989 at the University of Hamburg, Germany with the degree of staatsexamen (state exam).

His basic field of research interest is the relationship between cause and effect under many conditions, i. e. under conditions of quantum and relativity theory, in biomedical sciences, in philosophy et cetera.

Thus far, among his publications in physics, they are the refutation of Heisenberg's uncertainty principle ("Anti Heisenberg - Refutation of Heisenberg's uncertainty relation," American Institute of Physics Conference Proceedings, volume 1327, pp. 322-325, 2011), the refutation of Bell's theorem and the CHSH inequality ("Anti-bell - Refutation of bell's theorem," American Institute of Physics - Conference Proceedings, volume 1508, pp. 354-358, 2012). In another public, "The equivalence of time and gravitational field," Physics Procedia, volume 22, pp. 56-62, 2011). 